\title{
Multipath Exploitation in Emitter Localization for Irregular Terrains
}

\author{
Yaser DALVEREN ${ }^{1}$, Ali KARA ${ }^{2}$ \\ ${ }^{1}$ Dept. of Avionics, Atilim University, Kizilcasar Mahallesi, 06830 Incek Golbasi, Ankara, Turkey \\ ${ }^{2}$ Dept. of Electrical and Electronics Eng., Atilim Univ., Kizilcasar Mahallesi, 06830 Incek Golbasi, Ankara, Turkey
}

yaser.dalveren@atilim.edu.tr, ali.kara@atilim.edu.tr

Submitted October 17, 2018 / Accepted March 3, 2019

\begin{abstract}
Electronic Support Measures (ESM) systems have many operational challenges while locating radar emitter's position around irregular terrains such as islands due to multipath scattering. To overcome these challenges, this paper addresses exploiting multipath scattering in passive localization of radar emitters around irregular terrains. The idea is based on the use of multipath scattered signals as virtual sensor through Geographical Information System (GIS). In this way, it is presented that single receiver (ESM receiver) passive localization can be achieved for radar emitters. The study is initiated with estimating candidate multipath scattering centers over irregular terrain. To do this, ESM receivers' Angle of Arrival (AOA) and Time of Arrival (TOA) information are required for directly received radar pulses along with multipath scattered pulses. The problem then turns out to be multiple-sensor localization problem for which Time Difference of Arrival (TDOA)-based techniques can easily be applied. However, there is high degree of uncertainty in location of candidate multipath scattering centers as the multipath scattering involves diffuse components over irregular terrain. Apparently, this causes large localization errors in TDOA. To reduce this error, a reliability based weighting method is proposed. Simulation results regarding with a simplified $3 D$ model are also presented.
\end{abstract}

\section{Keywords}

Emitter localization, multipath, scattering center, virtual sensor, Time Difference of Arrival (TDOA)

\section{Introduction}

In many civilian and military applications, estimating the position of a source is a classical problem. In fact, this has been an issue of debate for many years. Especially in military applications, it is very important to locate and track threats. Threats can be considered as a source of emissions or emitters. Typically, emitter position is determined by using different sources of information and sensor measurements. Conventional localization schemes exploit the signals received by a multi-platform system or multiple sensor system [1]. These signals are considered to be propagated through line-of-sight (LOS) or direct paths from the emitter. However, multipath scattering adversely affects the performance of the localization schemes. To suppress the multipath effects, multipath mitigation techniques have been proposed [2]. On the contrary, exploiting multipath scattering has gained many interests in the last decades [3-10]. The works in [3-5] are based on the measurements from a multiple sensor system. On the other hand, studies in [6-9] have been proposed as single sensor localization system. Specifically, these studies can be used in indoor or urban applications. As an outdoor context, the work in [10] attempts to solve the source localization problem in naval applications. The proposed method is based on angle of arrival (AOA) and time difference of arrival (TDOA) measurements. It is tested in both real and simulated data to show its potential.

Therefore, solving emitter localization problem in outdoor context by exploiting multipath with a single receiver has become interesting to investigate. Certainly, new approaches or methods by considering realistic environment conditions should be taken into account. In this way, this study proposes a novel method for passive localization of radar emitters around irregular terrains such as islands. It is based on exploiting multipath scattering by using only a single receiver. Unlike other studies [6-10], multipath exploitation employed in this method involves diffuse scattering over irregular terrain. The proposed method begins with estimating candidate multipath scattering centers. To this end, firstly, AOA and Time of Arrival (TOA) information of multipath components are used. Indeed, this leads to determine an uncertainty region over the irregular terrain. Candidate multipath scattering centers over these regions are then estimated. To do this, Geometric Optic (GO) based search algorithm [11] along with Geographical Information Systems (GIS) can be employed. However, this may cause high degree of uncertainty in positioning of candidate multipath scattering centers. For this reason, knowledge of probability that a candidate multipath scattering center becomes a virtual sensor motivates the use of reliability-based weighted average method. Once the positions of virtual sensors are determined, the problem turns 
out to be multiple-sensor localization problem. Thus classical TDOA-based techniques can easily be applied.

The rest of the paper is organized as follows; problem statement is described in Sec. 2. The theoretical background of the study is discussed in Sec. 3. The proposed method for the solution of emitter localization problem is presented in Sec. 4. In Sec. 5, simulation results are provided. Discussions are given in Sec. 6, and followed by conclusion drawn in the last section.

\section{Problem Statement}

An Electronic Support Measures (ESM) system mainly consists of a wide-band intercept receiver and signal identification components. It cooperates with electronic warfare systems. It performs the tasks of detection, identification and classification of radar emitters in operational environments. In dense scattering environments, radiated signals from radar emitters are collected by the intercept receiver of the ESM system. These signals are received through the direct path (LOS) and multipath. Usually, multipath signals cause difficulties in discriminating radar emitters. This is because they have same parameters of radar emitters in the radar field of view. Indeed, received signals and their multipath scattered equivalents are processed at the ESM system as two different radar emitters. This entails increasing computational load at the ESM system.

This study aims to exploit multipath scattering over irregular terrain for locating of radar emitters. For this purpose, a simplified two-dimensional (2D) problem geometry (ignoring the altitude) is shown in Fig. 1.

In Fig. 1, radar emitter $\left(\mathbf{u}^{\circ}\right)$ with a beamwidth $\theta_{\mathbf{u}^{\mathrm{o}}}$, and an intercept receiver $\left(\mathbf{s}_{0}{ }^{\circ}\right)$ are indicated for illustration. Multipath scattering centers $\left(\mathbf{s}_{m}\right.$, where $m=1, \ldots, M$, and $M$ is the number of shaded regions) are also shown as circles. Firstly, it is necessary to define the boundary of the emitter's region. This will facilitate the solution of the localiza-

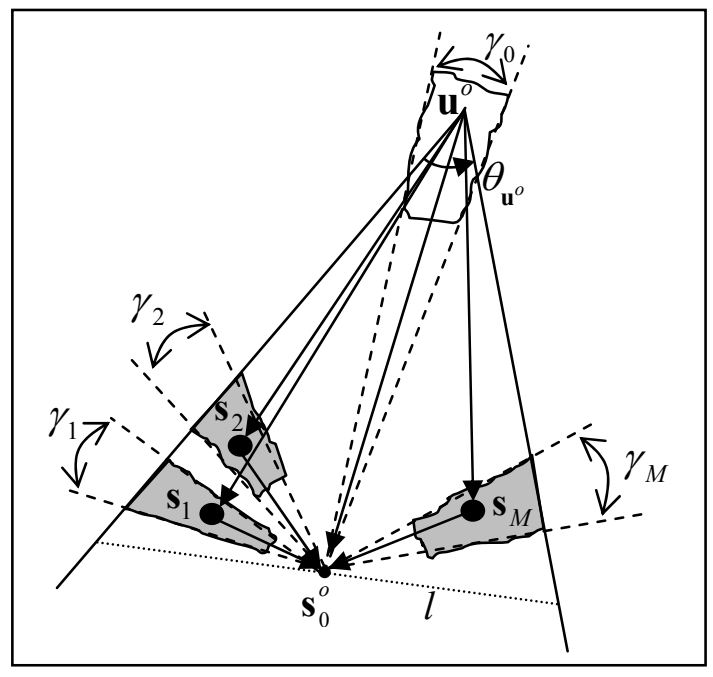

Fig. 1. Emitter localization problem. tion problem. To define the boundary of the region, some frequently used radar types and their parameters are utilized. All of this information can be reached from online sources [12]. It should be noted that peak transmitting power of these radar types is usually known. In addition, received power of the radar pulses is measured by intercept receiver. Then, the distance between intercept receiver and radar emitter can be estimated. This holds in case of free space (LOS). Due to imperfections either in the radar transmitter or intercept receiver, the distance has an error. It corresponds to the width of the region in perpendicular to the direction to intercept receiver.

On the other hand, intercept receiver is also able to measure the AOA of radar pulses with an error margin $\left(\gamma_{0}\right.$ and $\gamma_{m}$ ). The error margin in AOA is typically between $2^{\circ}$ and $8^{\circ}$ [12]. Therefore, the width of boundary of the emitter's region is specified by the error margin. This is also the case for the shaded regions shown in Fig. 1. These regions correspond to scattering regions over irregular terrain (usually rough surface) where the multipath scattering centers can be located. According to the figure these regions should be determined in the radar cell $(l)$. It represents the radius of the radar cell in a volumetric region. However, as 2D problem is considered, it corresponds to the projection on the ground. The outer boundary of the shaded regions can be then determined easily. The TDOA between directly received pulse and multipath pulse can also be measured by intercept receiver. This measurement must be greater than the pulse width. Otherwise, directly received pulses and multipath pulses are overlapped in time. Thus, multipath pulses cannot be exploited. If the condition is satisfied, a minimum distance from the intercept receiver to the inner boundary of the shaded regions can be determined. This distance can be only achieved when the TDOA measurements are expressed in terms of range differences. At this point, it is widely known that the time difference is easily converted to range difference by multiplying it with the speed of light $(c)$.

As the shaded regions are considered as irregular terrains, multipath pulses are exposed to diffuse scattering. The local region from which the multipath pulses are scattered will be then a multipath scattering center. However, an uncertainty is expected in position of multipath scattering centers. This may also cause large localization errors when the uncertainty is at high degree. To increase the accuracy, this study provides a novel method. It proposes that localization error could be minimized if the multipath scattering centers are accurately estimated. Details are described in the following sections.

\section{Theoretical Background}

This section is devoted to building the relationship between the transmitting signal energy, the received signal energy and the proposed method. In this way, the probability that a segment of a shaded region is the scattering center can be determined. 


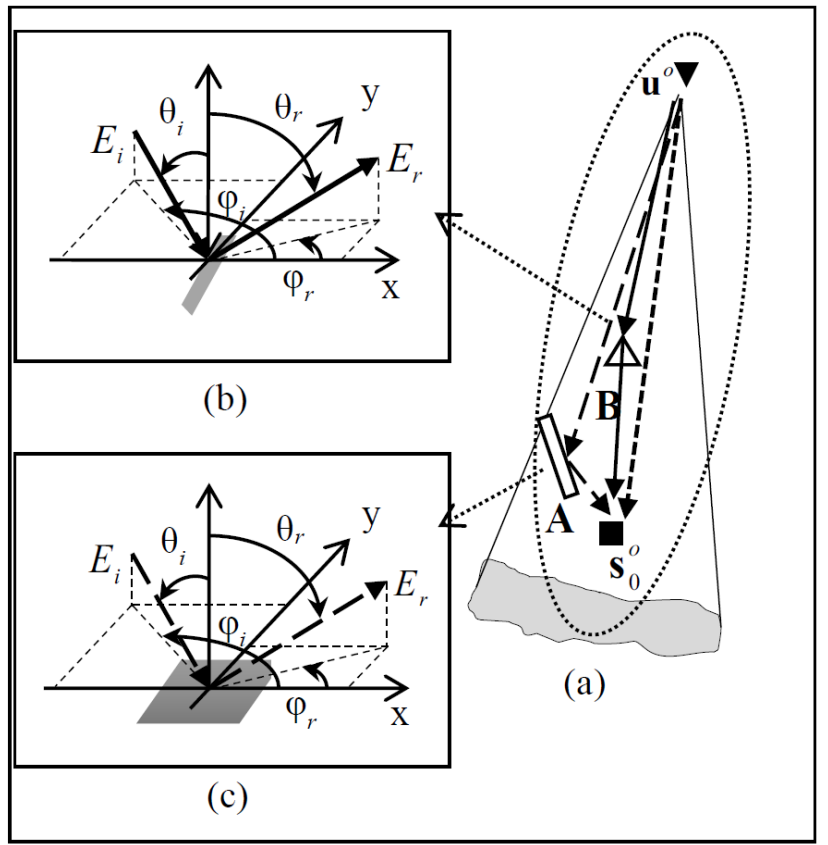

Fig. 2. (a) Propagation model (top view): ellipse denotes Fresnel zone for a given propagation path. (b) Diffraction in $3 \mathrm{D}$ at point $\mathrm{B}$. (c) Reflection from planar surface in $3 \mathrm{D}$ at point $\mathrm{A}$.

In this study, some propagation mechanisms such as reflection and diffraction should be accounted due to irregularities of the terrain. In fact, such mechanisms from irregular terrains are basic mechanisms of wave propagation. An illustrative example which shows a propagation model considered in this study is given in Fig. 2.

In Fig. 2(a), three waves emitted from radar emitter are arrived to the intercept receiver. The dominant wave directly arrives at intercept receiver through the LOS path. Other waves are reflected or diffracted as they interact with an obstruction. The obstructions are denoted as $\mathbf{A}$ and $\mathbf{B}$. Fresnel zone generated between radar emitter and intercept receiver provides a way of estimating how these obstructions may cause path loss for a particular link. It is also evident that these obstructions will fall inside of the Fresnel zone. These obstructions will be also inside the vertical plane containing the radar emitter and intercept receiver due to the specific cases used in determining shaded regions as discussed in previous section. Thus, both reflected and diffracted wave from the obstructions will be measurable at intercept receiver. At obstruction $\mathbf{A}$, the surface from which the incident wave is reflected is assumed to be locally flat. This will yield specular reflection which is also known as the Fresnel approximation. In addition to specular reflection, diffraction is also expected to occur due to edge shaped obstacles along or near the LOS path. This is shown at obstruction $\mathbf{B}$ in the figure.

\subsection{Propagation Loss Due to Reflection}

Reflection from local planar surface is shown in Fig. 2(c). In the figure, $i$ and $r$ refer to incident and reflected fields, respectively. The directions of the waves are specified by polar $(\theta)$ and azimuth angles $(\varphi)$. The wave can be depolarized into its vertical and horizontal E-field components. Superposition may be then applied to determine reflected waves [13]. The vertical and horizontal field components may be related by

$$
\left[\begin{array}{c}
E_{\mathrm{H}}^{\mathrm{r}} \\
E_{\mathrm{V}}^{\mathrm{r}}
\end{array}\right]=\mathbf{R}^{\mathrm{T}} \mathbf{D}_{\mathrm{C}} \mathbf{R}\left[\begin{array}{c}
E_{\mathrm{H}}^{\mathrm{i}} \\
E_{\mathrm{V}}^{\mathrm{i}}
\end{array}\right]
$$

where $E_{\mathrm{H}}{ }^{\mathrm{r}}$ and $E_{\mathrm{V}}{ }^{\mathrm{r}}$ are the depolarized field components in the horizontal and vertical directions, respectively. $E_{\mathrm{H}}{ }^{\mathrm{i}}$ and $E_{\mathrm{V}}{ }^{\mathrm{i}}$ are the horizontally and vertically polarized components of the incident wave. $\mathbf{R}$ is a transformation matrix which maps vertical and horizontal polarized components to the components which are perpendicular and parallel to the plane of incidence. The matrix $\mathbf{R}$ is given by

$$
\mathbf{R}=\left[\begin{array}{cc}
\cos \phi & \sin \phi \\
-\sin \phi & \cos \phi
\end{array}\right]
$$

where $\phi$ is the angle between the two sets of axes (horizontal and vertical axis). In Fig. 2(c), this angle corresponds to $\theta_{\mathrm{i}}$ when $\mathrm{x}-\mathrm{z}$ plane is considered. If $\mathrm{x}-\mathrm{y}$ plane is considered, then it corresponds to $\varphi_{\mathrm{i}}$.

The depolarization matrix $\mathbf{D}_{\mathrm{C}}$ in (1) is given by

$$
\mathbf{D}_{\mathrm{C}}=\left[\begin{array}{cc}
\Gamma_{\perp} & 0 \\
0 & \Gamma_{\|}
\end{array}\right]
$$

where $\Gamma_{\perp \perp}$ and $\Gamma_{\|}$are corresponded to Fresnel reflection coefficient for perpendicular and parallel polarizations, respectively. In general, Fresnel reflection coefficient depends on the wave polarization, angle of incidence, and the frequency of the propagating wave.

\subsection{Diffraction Loss}

The simplest and very common way to estimate diffraction loss is to treat the obstructing object as a knifeedge. In order to illustrate this situation, consider a 2D scenario consisted of a transmitter and a receiver at points $T_{\mathrm{x}}$ and $R_{\mathrm{x}}$ as shown in Fig. 3. A knife-type obstacle with height $h$ is placed between them at a distance $d_{1}$ from the $T_{\mathrm{x}}$, and $d_{2}$ from the $R_{\mathrm{x}}$.

The amount of diffraction loss is proportional to the level of obstruction of Fresnel zones by the obstacles.

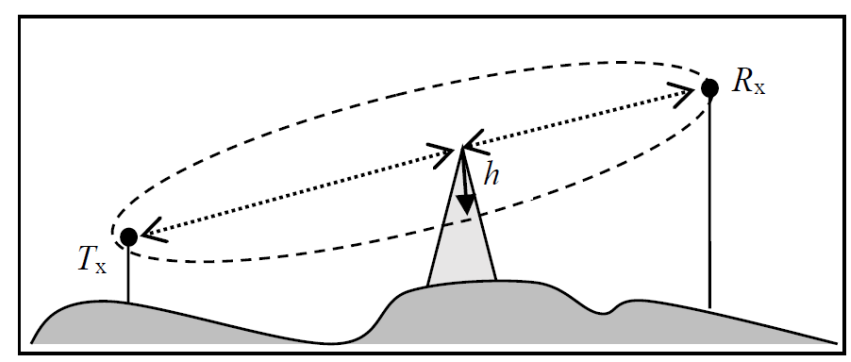

Fig. 3. Illustration of Fresnel zone for a knife-edge diffraction scenario. 
Historically, optics based approaches, such Geometric Optics (GO), might also estimate diffraction loss. GO might work well if there is no blockage or obstruction in the environment [14]. However, if there is an obstacle in the Fresnel zone as shown in Fig. 3, Geometrical Theory of Diffraction (GTD), as an extension to GO, can be successfully employed to calculate diffraction loss over the links. For multiple diffraction scenarios, there are also several approaches that have been proposed in the literature [15].

By considering a knife-edge model along with GTD, the E-field strength is given by [13]

$$
\frac{E_{\mathrm{d}}}{E_{\mathrm{o}}}=F(v)=\frac{(1+\mathrm{j})}{2} \int_{v}^{\infty} \exp \left(\left(-\mathrm{j} \pi t^{2}\right) / 2\right) \mathrm{d} t
$$

where $E_{\mathrm{d}}$ is the E-field strength of diffracted wave, $E_{\mathrm{o}}$ is the free space field strength, $F(v)$ is the complex Fresnel integral which is a function of Fresnel diffraction parameter $v$. It is defined as

$$
v=h \sqrt{\frac{2\left(d_{1}+d_{2}\right)}{\lambda d_{1} d_{2}}}
$$

where $\lambda$ is the operational wavelength. $F(v)$ is estimated by using tables or values according to $v$. Diffraction loss $\left(L_{\mathrm{d}}\right)$ or in other term diffraction gain $\left(G_{\mathrm{d}}=-L_{\mathrm{d}}\right)$ is then calculated by

$$
G_{\mathrm{d}}(\mathrm{dB})=20 \log |F(v)| .
$$

Graphical representation of knife-edge diffraction gain as a function of $v$ is also shown in Fig. 4.

\subsection{Total Path Loss}

Obviously, a propagation model of single LOS path is inaccurate for predicting the path loss characteristics observed over the environment. In the presence of obstructions as shown in Fig. 2(a), reflection and diffraction concepts of previous sub-sections should be integrated. With this viewpoint, the total path loss is consisted of the freespace path loss $\left(L_{\mathrm{f}}\right)$, the reduction in the received field due

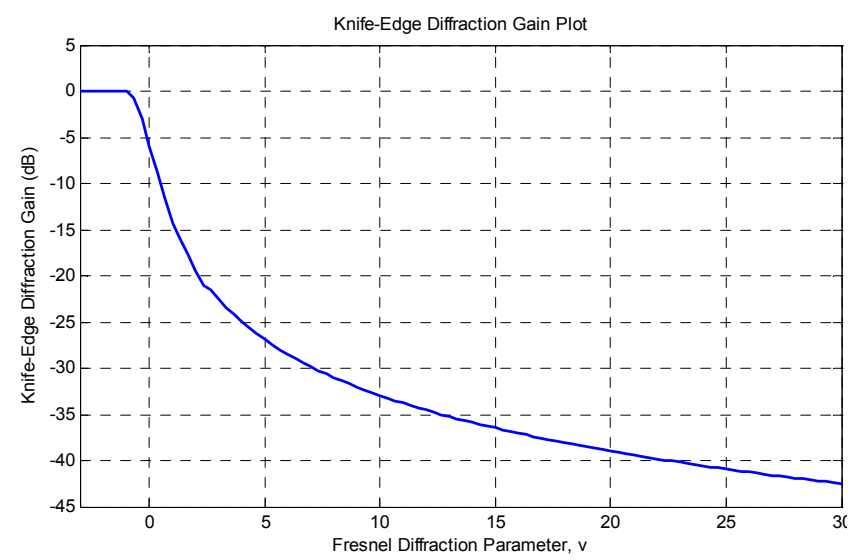

Fig. 4. Knife-edge diffraction gain $\left(G_{\mathrm{d}}\right)$ as a function of $v$. to reflection $\left(L_{\mathrm{r}}\right)$, and the loss due to diffraction $\left(L_{\mathrm{d}}\right)$. Total path loss of the propagation model considered in this study is then expressed as

$$
P L(\mathrm{~dB})=L_{\mathrm{f}}+L_{\mathrm{r}}+L_{\mathrm{d}} .
$$

When antenna gains are excluded, then free space path loss is calculated by

$$
L_{\mathrm{F}}(\mathrm{dB})=20 \log \left[\frac{\lambda}{(4 \pi) d}\right]
$$

where $\lambda$ is the wavelength, and $d$ is the distance between the receiver and the transmitter.

In practice, specular reflection is more likely to occur at typical radar frequencies. Particularly, when knife-edge obstacles are far away from the LOS path, reflection becomes dominant in estimating the received signal power. This is because diffracted signal power by the knife-edge in the direction can be ignored. For this reason, diffracted signals from these obstacles will not be measurable by the system. The total path loss will be then only the product of $L_{\mathrm{f}}$ and $L_{\mathrm{r}}$. Therefore it can be concluded that the factors for calculating the total path loss is dependent on the localization scenario.

Therefore, the procedures described above will be employed in an efficient ray tracing algorithm as presented in [16]. Although the main purpose of this study is not to provide such an algorithm, it is necessary to discuss its structure. In the first step of the algorithm, the region shown in Fig. 1 in which the emitter may be located should be split up into segments. These segments can be taken as possible emitter locations. This will provide to construct the links between the multipath scattering centers and possible emitter locations. To determine the multipath scattering centers, the procedure described in the next section can be followed. Hence, the loss of each links will also be estimated by applying GO or GTD based wave propagation techniques. These losses can be then used in the proposed method. All these steps can be implemented by taking into account the digital terrain elevation data (DTED) [17]. In order to achieve the digital terrain data, GIS should be used. Here, it is important to note that spatial data visualized in a GIS comes in vector and/or raster format. An open source tool can be then used to split the vector or raster dataset into separate files which are based on the DTED tiling scheme. It is widely known that only three levels of the DTED tiling scheme are available for usage: level 0 with 900 m resolution, level 1 with 90 m resolution and level 2 with $30 \mathrm{~m}$ resolution. Besides, there are other levels such as level 3, 4, and 5 that have been proposed for higher resolution. However, these levels have not been standardized yet. It should be also noted that the higher level of DTED tiling scheme plays a critical role to gather precise data to be used in ray tracing algorithm. Therefore, DTED as a standard of digital dataset that consists of a matrix of terrain elevation values can be provided. 


\section{Proposed Method}

This section presents the proposed method to achieve a solution for the given problem in Sec. 2. To describe the method, multipath scattered pulses that are collected by the intercept receiver of the ESM system should be simply illustrated as shown in Fig. 5.

In the figure, $\tau$ denotes pulse width, $t_{0}$ denotes the TOA of directly received pulse, and $t_{m}$ denotes TOA of multipath scattered pulses where $m=1, \ldots, M$. TDOA of directly received pulse and multipath scattered pulses are denoted by $t_{0 m}$. In addition, $P_{0}$ denotes received power of directly received pulse while $P_{m}$ denotes received power of multipath scattered pulses.

The initial stage of the proposed method consists of determining shaded regions. This can be accomplished by $t_{0 m}$ along with $\gamma_{m}$ as discussed in Sec. 2. The size of these regions is dependent on the parameters of radar transmitter and intercept receiver along with the geometry of the problem. In practice, these regions can be either narrower or wider depending on the geometry of the problem. For the case of narrower shaded regions, the multipath scattering centers could be placed at the center of these regions. In this case, the center of the shaded region is assumed as a virtual sensor. Source localization techniques using TDOA measurements in the presence of sensor position errors could then easily be adapted to solve the localization problem. Unfortunately, accuracy of TDOA methods is very sensitive to sensor position errors. However, the solution can be acceptable as long as the error in sensor position is tolerable [18-20]. The accuracy of these TDOAbased localization methods has been also evaluated in [21]. It has shown that improved-two-step weighted least square (improved-TSWLS) method [20] is performed as the most efficient estimator at high sensor position errors. For this reason, it has been decided to use in the proposed method.

On the other hand, for the case of wider shaded regions, the uncertainty in the multipath scattering centers will become large. This may adversely affect the localization accuracy. To overcome this problem, segmentation is adopted to split the shaded regions up to equivalent segments. These segments are then assumed as candidate multipath scattering centers. Among the candidates, multipath scattering centers to be used in emitter localization are required to determine. In fact, multipath scattering centers can be determined from the segments which are electromagnetically visible to both the radar emitter and intercept receiver. Therefore, likelihood of the segments from which the multipath scattering takes place are calculated.

For the case of reflection, the maximum loss due to reflection $\left(L_{\mathrm{r}_{\max }}\right)$ depends on the minimum signal power of the reflected pulses measured by the intercept receiver (corresponding to the minimum detectable signal at the ESM system). Suppose that $N_{M}$ is the number of segment of the $M^{\text {th }}$ shaded region that is electromagnetically visible

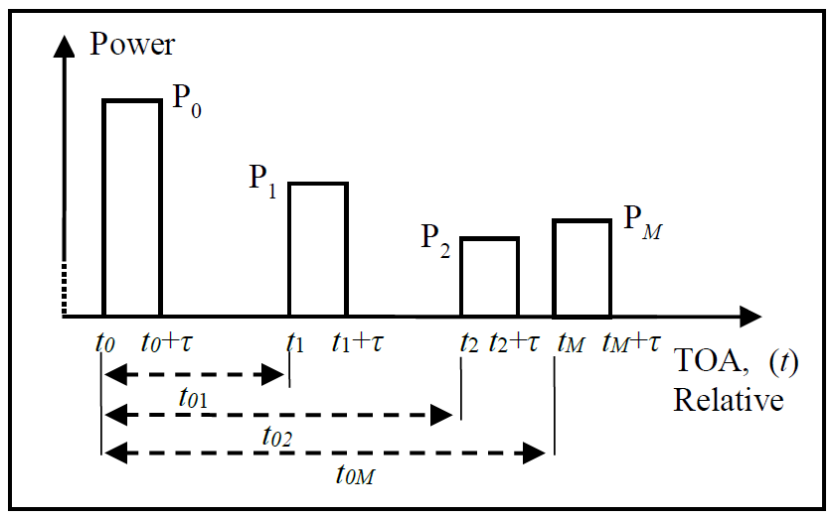

Fig. 5. TOA of multipath scattered pulses.

to both the radar emitter and intercept receiver, and the loss for the $i^{\text {th }}$ segment out of $N_{M}$ is $L_{\mathrm{r}_{i}}$. If each of $L_{\mathrm{r}_{i}}$ is normalized by $L_{\mathrm{r}_{\max }}$, then the likelihood of the segment $i$ being the source of the multipath scattered pulse, $p\left(L_{\mathrm{r}_{i}}\right)$, can be calculated by classical probability [22]. This can be expressed as

$$
p\left(L_{\mathrm{r}_{i}}\right)=\frac{L_{\mathrm{r}_{i}} / L_{\mathrm{r}_{\max }}}{\sum_{j=1}^{N_{M}} L_{\mathrm{r}_{j}} / L_{\mathrm{r}_{\max }}}, \quad i=1, \ldots, N_{M} .
$$

For the case of diffraction, an illustrative example has been used to determine a threshold as a function of $v$ [23]. It has been shown that a $40 \mathrm{~dB}$ threshold can be determined for the maximum diffraction loss $\left(L_{\mathrm{d}_{\max }}\right)$ of the knife-edge model. However, some other models encompassing physical insights of the problem for calculating $L_{\mathrm{d}_{\max }}$ can be adapted. Note that the diffraction loss should be determined for both links, from the radar emitter to the $i^{\text {th }}$ segment $\left(L_{\mathrm{Ts}_{i}}\right)$, and from the $i^{\text {th }}$ segment to the intercept receiver $\left(L_{\mathrm{s}_{\mathrm{R}} \mathrm{R}}\right)$. Using (9), the likelihoods of these links can be easily calculated by replacing $L_{\mathrm{r}_{\max }}$ with $L_{\mathrm{d}_{\max }}$, and $L_{\mathrm{r}_{i}}$. with both $L_{\mathrm{Ts}_{i}}$ and $L_{\mathrm{s}_{i} \mathrm{R}}$. If the links are considered as independent of each other, the likelihood of the $i^{\text {th }}$ segment out of $N_{M}, p\left(L_{\mathrm{d}_{\mathrm{i}}}\right)$, can be calculated by means of independence of random events in probability [22]

$$
p\left(L_{\mathrm{d}_{i}}\right)=\frac{p\left(L_{\mathrm{Ts}_{i}}\right) \cdot p\left(L_{\mathrm{s}_{i} \mathrm{R}}\right)}{\sum_{j=1}^{N_{M}} p\left(L_{\mathrm{Ts}_{j}}\right) \cdot p\left(L_{\mathrm{s}_{j} \mathrm{R}}\right)}
$$

where $\mathrm{p}\left(L_{\mathrm{Ts}_{i}}\right)$ and $p\left(L_{\mathrm{s}_{i} \mathrm{R}}\right)$ are the likelihoods of the links from radar emitter to $i^{\text {th }}$ segment, and from $i^{\text {th }}$ segment to intercept receiver, respectively. Therefore, either $p\left(L_{\mathrm{r}_{\mathrm{j}}}\right)$ or $p\left(L_{\mathrm{d}_{\mathrm{i}}}\right)$ can be attributed to the likelihood of the $i^{\text {th }}$ segment being a virtual sensor, $p\left(\mathbf{s}_{i}\right)$, as given in the following

$$
p\left(\mathbf{s}_{i}\right)= \begin{cases}p\left(L_{\mathrm{r}_{i}}\right), & \text { if reflection occurs } \\ p\left(L_{\mathrm{d}_{i}}\right), & \text { if diffraction occurs. }\end{cases}
$$


In this study, it is worth noting that propagation mechanisms over the shaded regions are considered to be independent from each other. In other words, each of shaded regions is assumed to have different propagation characteristics. On the other hand, it is evident that there may be more than one segment being a virtual sensor for any particular shaded region. Thus, all the combinations of segments selected from $M$ shaded regions must be taken into account. The number of combinations, $k$, can be calculated by

$$
k=\prod_{i=1}^{M} N_{i} .
$$

As the likelihoods are independent of each other, the likelihood of the each combination, $p\left(\hat{\mathbf{s}}_{k}\right)$, can be expressed by considering reliability of series system [22] as

$$
\begin{aligned}
p\left(\hat{\mathbf{s}}_{1}\right) & =p\left(\mathbf{s}_{i 1}\right) \cdot p\left(\mathbf{s}_{i 2}\right) \cdot \ldots \cdot p\left(\mathbf{s}_{i M}\right) \\
& \vdots \\
p\left(\hat{\mathbf{s}}_{k}\right) & =p\left(\mathbf{s}_{N_{1} 1}\right) \cdot p\left(\mathbf{s}_{N_{2} 2}\right) \cdot \ldots \cdot p\left(\mathbf{s}_{N_{M} M}\right) .
\end{aligned}
$$

In (13), $p\left(\mathbf{s}_{i M}\right)$ is the likelihood of the $i^{\text {th }}$ segment being a virtual sensor of the $M^{\text {th }}$ shaded region which can be calculated by using (11).

In the last stage of the proposed method, the aim is to provide unique solution by averaging all emitter positions along with the likelihoods of each segment being a virtual sensor. To do this, firstly, virtual sensor groups have to be stored due to the fact that there may be more than one virtual sensor for any particular shaded region. This suggests that there may be more than one estimated emitter's position. The reason is that each combination of virtual sensors selected from $M$ shaded regions would give a unique emitter's position. Similar to (13), sensor groups can be easily stored. Yet, each group has to be consisted of intercept receiver's position $\left(\mathbf{s}_{0}{ }^{\circ}\right)$ and virtual sensor positions in order from $M$ different shaded regions. Then, let us define a matrix $\mathbf{S}=\left[\hat{\mathbf{s}}_{1}, \hat{\mathbf{s}}_{2}, \ldots, \hat{\mathbf{s}}_{k}\right]^{\mathrm{T}}$. In this matrix, $\hat{\mathbf{s}}_{k}$ represents the $k^{\text {th }}$ virtual sensor group. Hence, $\mathbf{S}$ becomes

$$
\mathbf{S}=\left[\begin{array}{ccccc}
\mathbf{s}_{0}^{\mathrm{o}} & \mathbf{s}_{i 1}^{\mathrm{o}} & \mathbf{s}_{i 2}^{\mathrm{o}} & \ldots & \mathbf{s}_{i M}^{\mathrm{o}} \\
& & \vdots & & \\
\mathbf{s}_{0}^{\mathrm{o}} & \mathbf{s}_{N_{1} 1}^{\mathrm{o}} & \mathbf{s}_{N_{2} 2}^{\mathrm{o}} & \ldots & \mathbf{s}_{N_{M} M}^{\mathrm{o}}
\end{array}\right] .
$$

In (14), $\mathbf{s}^{\mathrm{o}}{ }_{i M}$ denotes the $i^{\text {th }}$ virtual sensor position from the $M^{\text {th }}$ shaded region. Therefore, $k$ different emitter positions can be determined for each group in $\mathbf{S}$ by applying improved-TSWLS method [20]. In doing so, another matrix covering the emitter positions such as $\mathbf{u}=\left[\mathbf{u}_{1}, \mathbf{u}_{2}, \ldots, \mathbf{u}_{k}\right]^{\mathrm{T}}$ can also be defined. The position of the radar emitter, $\hat{\mathbf{u}}$, can be then estimated by weighting each row of $\mathbf{u}$ with the corresponding likelihood of each row of S. This corresponds to weighted average calculation, and it can be expressed as

$$
\hat{\mathbf{u}}=\sum_{i=1}^{k} p\left(\hat{\mathbf{s}}_{i}\right) \cdot \mathbf{u}_{i} .
$$

\section{Simulations}

In this section, simulation results are presented to promote the theoretical development of the proposed method. The simulation scenario containing the parameters is listed in Tab. 1.

By considering Sec. 2 along with the parameters of the simulation scenario, localization geometry in 2D was obtained. To perform the proposed method, obtained shaded regions were approximated as polygons for the sake of simplicity. As discussed in the previous section, one of the approaches for determining emitter location is to place virtual sensors at the center of the shaded regions. This approach, however, would provide efficient results if narrower shaded regions were obtained. This is also the case rarely encountered in practice. It should be mentioned that the size of shaded regions obtained from the given simulation scenario was relatively wide. Segmentation was then performed on these polygons according to DTED format for level 1 and 2. These levels correspond to $90 \mathrm{~m} \times 90 \mathrm{~m}$ and $30 \mathrm{~m} \times 30 \mathrm{~m}$ segments, respectively. For an accurate performance evaluation of the proposed method, $45 \mathrm{~m} \times 45 \mathrm{~m}$ and $22.5 \mathrm{~m} \times 22.5 \mathrm{~m}$ segments were also considered in segmentation. Virtual sensor positions were then determined from all shaded regions for the specified segment sizes. Therefore, for $M=4, N$ was determined as $1,4,9$, and 16 segments for $90 \mathrm{~m} \times 90 \mathrm{~m}, 45 \mathrm{~m} \times 45 \mathrm{~m}$, $30 \mathrm{~m} \times 30 \mathrm{~m}$, and $22.5 \mathrm{~m} \times 22.5 \mathrm{~m}$ sized segments, respectively. Here, it is worth noting that $M$ was limited to the lowest possible level in the simulations. This is due to the fact that a high number of multipath signals cannot be predicted in most practical cases. In addition, $N$ was fixed in all shaded regions for simplicity. As an illustration, all procedure described above for the shaded region of $\gamma_{1}{ }^{\circ}=58^{\circ}$ is shown in Fig. 6. On the other hand, the upper limit of virtual sensor position noise power $\left(\sigma_{\mathrm{S}}{ }^{2}\right)$ was determined according to the size of the segments. To do this, three-sigma rule was utilized. The upper limit was then calculated to be approximately $20 \mathrm{~dB}, 15 \mathrm{~dB}, 12 \mathrm{~dB}$ and $9 \mathrm{~dB}$ for $90 \mathrm{~m} \times 90 \mathrm{~m}, 45 \mathrm{~m} \times 45 \mathrm{~m}, 30 \mathrm{~m} \times 30 \mathrm{~m}$, and $22.5 \mathrm{~m} \times 22.5 \mathrm{~m}$ sized segments, respectively. Table 2 summarizes the all relation between the $N$, segment size and $\sigma_{\mathrm{S}}^{2}$.

Moreover, before performing the simulations, likelihoods of the segments were randomly assigned. This is because, the losses for each links were assumed to be provided by using a GTD-based ray tracing algorithm. In this context, two different cases were considered to assign the likelihoods: a) dominant case, b) non-dominant case. For the dominant case, only one segment being a source of multipath scattered signals was considered. For this reason, the greater likelihood was assigned to one of the segments 


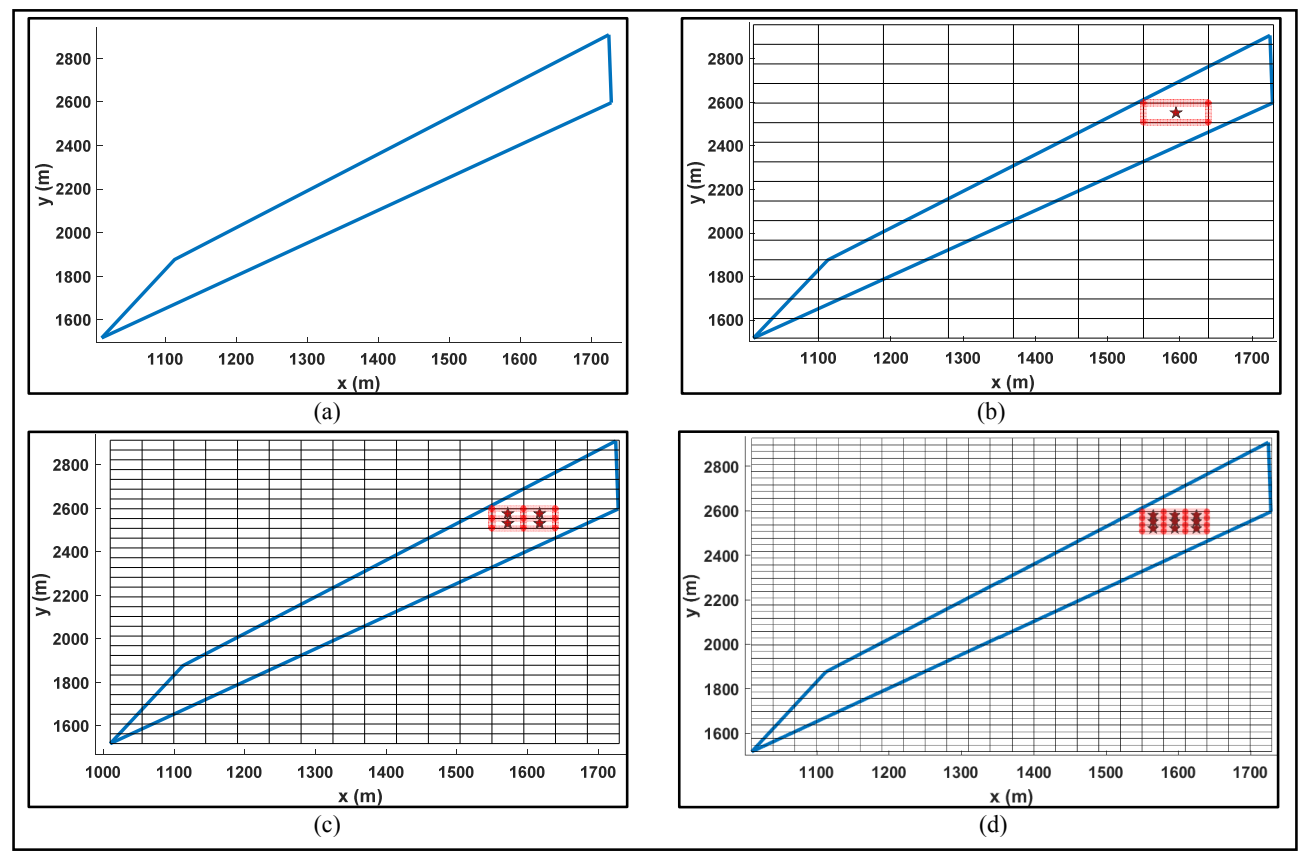

Fig. 6. (a) Illustration of a shaded region for $\gamma_{1}{ }^{\circ}=58^{\circ}$. (b) Segmentation for $90 \mathrm{~m} \times 90 \mathrm{~m}$. (c) Segmentation for $45 \mathrm{~m} \times 45 \mathrm{~m}$. (d) Segmentation for $30 \mathrm{~m} \times 30 \mathrm{~m}$.

\begin{tabular}{|c|c|}
\hline Parameters & Value \\
\hline $\mathbf{s}_{0}{ }^{\circ}$ & {$[0,0]^{\mathrm{T}} \mathrm{m}$} \\
\hline $\mathbf{u}^{\circ}$ & {$[900,75000]^{\mathrm{T}} \mathrm{m}$} \\
\hline$\theta_{\mathrm{u}^{\mathrm{o}}} ;$ Beamwidth & $2.7^{\circ}$ \\
\hline$\tau$, Pulsewidth & $1 \mu \mathrm{s}$ \\
\hline $\begin{array}{c}\gamma^{\mathrm{o}}=\left[\gamma_{0}^{\mathrm{o}}, \boldsymbol{\gamma}^{\mathrm{o}}{ }_{1}, \ldots, \gamma^{\mathrm{o}}{ }_{M}^{\mathrm{T}} ;\right. \\
\text { AOA of the pulses }\end{array}$ & $\gamma^{\mathrm{O}}=[89,58,120,227,304]^{\mathrm{T}}$ \\
\hline $\begin{array}{c}\Delta \gamma ; \\
\text { AOA error of the ESM system }\end{array}$ & $3^{\circ}$ \\
\hline $\begin{array}{c}\gamma=\left[\gamma_{1}, \ldots, \gamma_{M}\right]^{\mathrm{T}} \\
\text { Erroneous AOA }\end{array}$ & $\gamma=\gamma^{o} \pm(\Delta \gamma / 2)$ \\
\hline $\begin{array}{l}d_{0} ; \text { The distance } \\
\text { between } \mathbf{s}_{0}{ }^{\circ} \text { and } \mathbf{u}^{\circ}\end{array}$ & $\approx 75 \mathrm{~km}$ \\
\hline$\Delta d_{0} ;$ Range margin & $10 \mathrm{~km}$ \\
\hline
\end{tabular}

Tab. 1. Parameters regarding with the $2 \mathrm{D}$ localization geometry.

\begin{tabular}{|c|c|c|}
\hline Segment size & $N$ & $\sigma_{\mathrm{S}}{ }^{2}(\mathrm{~dB})$ \\
\hline $90 \mathrm{~m} \times 90 \mathrm{~m}$ & 1 & 20 \\
\hline $45 \mathrm{~m} \times 45 \mathrm{~m}$ & 4 & 15 \\
\hline $30 \mathrm{~m} \times 30 \mathrm{~m}$ & 9 & 12 \\
\hline $22.5 \mathrm{~m} \times 22.5 \mathrm{~m}$ & 16 & 9 \\
\hline
\end{tabular}

Tab. 2. Relation between the $N$, segment size and $\sigma_{\mathrm{S}}^{2}$.

while other likelihoods were distributed randomly to remained segments. For the non-dominant case, the likelihoods were equally assigned to the segments.

Furthermore, it was necessary to define the heights of the intercept receiver, the radar emitter and virtual sensors in order to obtain 3D localization geometry. To this end, the heights of the virtual sensors were specified between $550 \mathrm{~m}$ and $750 \mathrm{~m}$, and randomly assigned to the virtual sensor positions. The height of the intercept receiver was assigned as $600 \mathrm{~m}$ while the radar emitter was considered at

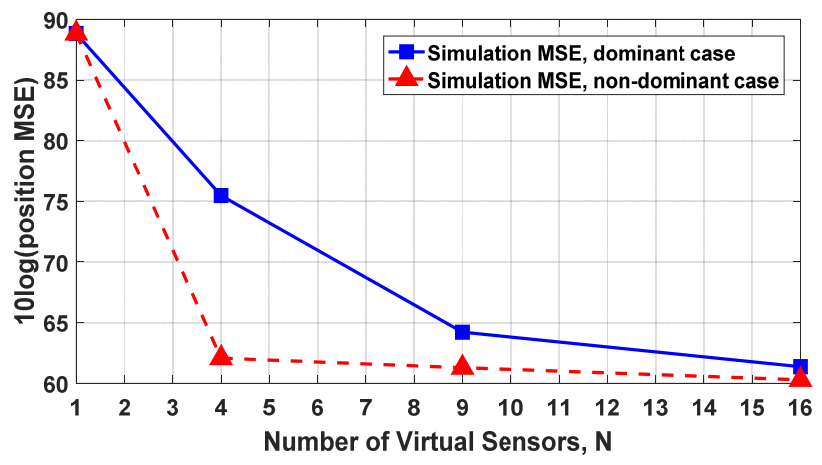

Fig. 7. Comparison of the MSE of the emitter position estimate for dominant and non-dominant case.

as $25 \mathrm{~m}$ in height. Accordingly, the intercept receiver position was located at $\mathbf{s}_{0}{ }^{\mathrm{O}}=[0,0,25]^{\mathrm{T}} \mathrm{m}$, and the radar emitter was assumed to be positioned at $\mathbf{u}^{\mathrm{o}}=[900,75000,600]^{\mathrm{T}} \mathrm{m}$. Therefore, to examine the accuracy, Mean Square Error (MSE) [18-21] of location estimate were obtained at $10^{3}$ ensemble runs by varying $N$. Results are shown in Fig. 7.

The simulation results can be evaluated in terms of $N$, and the distribution of the likelihoods. Particularly, the result for $N=1$ corresponds the improved-TSWLS method [20]. Accordingly, higher localization accuracy can be achieved by increasing the $N$ in the proposed method. However, this can be achieved only if a high-resolution digital elevation map is used in practice. This is because, it provides small-sized segments, and thus yields lower uncertainty in virtual sensor positions. Although increasing the $N$ has a positive impact on localization accuracy, the distribution of the likelihoods is still important parameter. According to the results, it is apparent that the distribution of the likelihood highly affects the localization accuracy. 


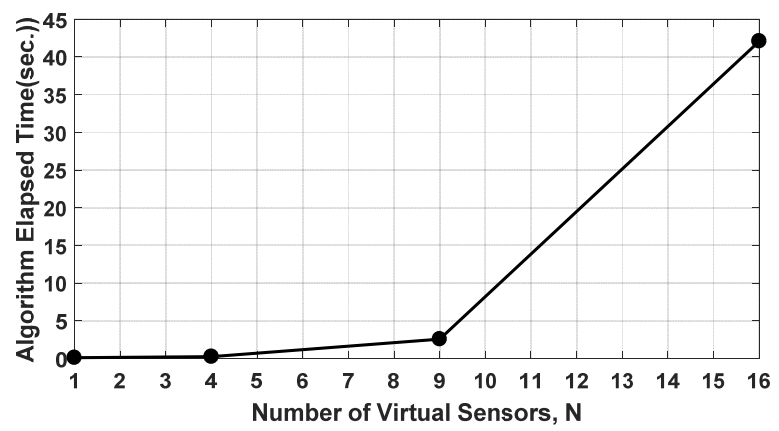

Fig. 8. Elapsed time of the proposed method.

On the other hand, algorithm elapsed time was also computed to evaluate the efficiency of the proposed method. To obtain computing times, MATLAB R2016b was used in computer system with configuration Intel $\mathbb{R}$ core $^{\mathrm{TM}}$ i7-7500U CPU, 8 GB RAM and 64bit operating system. Figure 8 shows the elapsed time of the algorithm with respect to $N$.

As it is shown, the algorithm elapsed time tends to increase with the increase of $N$. Particularly, when $N=9$, which is one of the extreme cases for the segmentation, the resulting complexity seems to be acceptable (nearly $2.5 \mathrm{~s}$ ). However, for another extreme case, when $N=16$, the complexity becomes worse. Thus, it can be concluded that the proposed method is time efficient when $N$ is not sufficiently large. Yet, it is worth noting that larger $N$ is not expected to be determined in practice. This makes it applicable in practice for relatively smaller $N$ (typically $N \leq 9$ ). Further discussion pertaining to simulation results and implementation of the proposed method in practice is provided in the next section.

\section{Discussions}

The previous section includes some simulations for analyzing the accuracy of the proposed method for a specific localization problem geometry. As can be inferred from the results, localization accuracy is significantly increased when there is a non-dominant distribution among the likelihoods. This results from the fact that the result of weighted averaging method converges to the mean of estimated emitter positions. For this reason, additional accuracy analysis should also be undertaken to clarify the performance evaluation. Even if the localization accuracy is analyzed in terms of MSE, further analysis such as Circular Error Probable (CEP) may also be used. Furthermore, position of the virtual sensors affects the localization accuracy especially under the poor location geometry which is the case of large geometric dilution of precision (GDOP). This case may give rise to significantly decrease in localization accuracy. Hence, further studies would be useful to enhance the proposed method by reducing the GDOP effect.

In practice, implementation of the proposed method relies on several factors. The important one is the ray tracing algorithm as discussed in Sec. 3. In this algorithm, all direct, reflected, and diffracted signals received by the receiver should be detected, and then all possible links should be determined. Especially for the case of diffraction, there needs to be a search method for the degree of clearance of Fresnel zones. Clearance of the Fresnel zone is then ensured by inspecting whether any obstacles exist or not in the link. When there is an obstacle in the link, diffraction loss should be accounted. Obviously, an efficient GTD-based ray tracing algorithm should be developed by using realistic obstruction models. Therefore, further studies would be required as identifying the likelihood of the segments from which the multipath scattering takes place is indicative for the localization accuracy. Another important factor is related to propagation mechanisms. In this study, shaded regions are assumed to have different propagation characteristics. In addition, only a single propagation mechanism for each of segments is considered. However, in practice, there may be different propagation mechanisms. In this case, total path loss should be accounted, and then additional approach should be developed to estimate the maximum loss. Indeed, this constitutes a part of ongoing research and will be investigated in future studies.

\section{Conclusion}

In the literature, there have been various approaches that provide solutions to the localizations of moving and stationary targets by a single receiver. However, there has been no evidence that localization of a stationary target by a single receiver by means of multipath can be achieved, especially in irregular terrains. This study proposes a method in locating of emitters through a single receiver (standard intercept receiver) by exploiting multipath from irregular terrains. Currently, all conventional intercept receivers, as considered to be a single sensor installed either stationary or a moving platform, do not have the capability of localization of emitters. With the proposed method, even a typical intercept receiver that provides only Angle of Arrival (AOA) \& Time of Arrival (TOA) information can achieve precise localization of emitters in such environments. In this way, multipath effects, as being one of the major problems in radio propagation, are used as an advantage in localization of emitters by available intercept receivers.

In summary, the proposed method is based on a novel idea that is the use of multipath scattering centers as virtual sensor in emitter localization. As the multipath scattering involves diffuse components over irregular terrain, there is an uncertainty in location of scattering centers. However, this adversely affects the localization accuracy. To reduce the effects of scattering center error, a new approach that is segmentation based weighted Time Difference of Arrival (TDOA) method has been proposed. Once virtual sensors are determined, reliability based weighting method is also adopted to estimate the emitter location. The accuracy and viability of the proposed method is shown by simulations. 


\section{Acknowledgments}

This work was supported by The Scientific and Technological Research Council of Turkey (TUBITAK) under the grant number $118 \mathrm{E} 157$.

\section{References}

[1] POISEL, R. Electronic Warfare Target Location Methods. $2^{\text {nd }}$ ed., London (UK): Artech House, 2005. ISBN: 9781608075232

[2] RIGLING, B. Urban RF multipath mitigation. IET Radar, Sonar \& Navigation, 2008, vol. 2, no. 6, p. 419-425. DOI: 10.1049/ietrsn:20080040

[3] LUI, K. W., SO, H. C. Range-based source localisation with pure reflector in presence of multipath propagation. IEEE Electronics Letters, 2010, vol. 46, no. 13, p. 957-958. DOI: $10.1049 / \mathrm{el} .2010 .3431$

[4] SEOW, C. K., TAN, S. Y. Localization of omni-directional mobile device in multipath environments. Progress In Electromagnetics Research, vol. 2008, no. 85, p. 323-348. DOI: 10.2528/PIER08090302

[5] SHEN, Y., WIN, M. Z. On the use of multipath geometry for wideband cooperative localization. In Proceedings of the IEEE Global Telecommunications Conference. Honolulu (USA), 2009. DOI: 10.1109/GLOCOM.2009.5425798

[6] MEISSNER, P., WITRISAL, K. Multipath-assisted single-anchor indoor localization in an office environment. In Proceedings of the IEEE $19^{\text {th }}$ International Conference on Systems, Signals and Image Processing (IWSSIP). Vienna (Austria), 2012, p. 22-25. ISBN: 978-3-200-02328-4

[7] MUQAIBEL, A. H., AMIN, M. G., AHMAD, F. Target localization with a single antenna via directional multipath exploitation. International Journal of Antennas and Propagation, 2015. DOI: $10.1155 / 2015 / 510720$

[8] O'CONNOR, A., SETLUR, P., DEVROYE, N. Single-sensor RF emitter localization based on multipath exploitation. IEEE Transactions on Aerospace and Electronic Systems, 2015, vol. 51, no. 3, p. 1635-1651. DOI: 10.1109/TAES.2015.120807

[9] SETLUR, P., SMITH, G. E., AHMAD, F., AMIN, M. G. Target localization with a single sensor via multipath exploitation. IEEE Transactions on Aerospace and Electronic Systems, 2012, vol. 48, no. 3, p. 1996-2014. DOI: 10.1109/TAES.2012.6237575

[10] GIACOMETTI, R., BAUSSARD, A., JAHAN, D., et al. Localization of radar emitters from a single sensor using multipath and TDOA-AOA measurements in a naval context. In Proceedings of the IEEE 24th European Signal Processing Conference (EUSIPCO). Budapest (Hungary), 2016, p. 692-696. DOI: 10.1109/EUSIPCO.2016.7760337

[11] KAPUSUZ, K. Y., KARA, A. Determination of scattering center of multipath signals using geometric optics and Fresnel zone concepts. Engineering Science and Technology, 2014, vol. 17, no. 2 , p. $50-57$. DOI: $10.1016 /$ j.jestch. 2014.03 .004

[12] JANE'S DEFENCE WEEKLY. [Online] Cited 2018-10-16 Available at: http://www.janes.com/defence/janes-defence-weekly

[13] RAPPAPORT, T. S. Wireless Communications - Principles and Practice. New Jersey (USA): Prentice Hall, 1996. ISBN: 9780780311671
[14] BERTONI, H. L. Radio Propagation for Modern Wireless Systems. London (UK): Pearson Education, 1999. ISBN: 0130263737

[15] KARA, A., BERTONI, H. L., YAZGAN, E. Limit and application range of the slope-diffraction method for wireless communications. IEEE Transactions on Antennas and Propagation, 2003, vol. 51, no. 9, p. 2512-2514. DOI: 10.1109/TAP.2003.816389

[16] TABAKCIOGLU, M. B. A top-down approach to S-UTD-CH model. Applied Computational Electromagnetics Society Journal, 2017, vol. 32 , no. 7 , p. 586-592. ISSN: $1054-4887$

[17] NATIONAL GEOSPATIAL-INTELLIGENCE AGENCY Performance Specification Digital Terrain Elevation Data (DTED). 45 pages. [Online] Cited 2019-02-01. Available at: https://dds.cr.usgs.gov/srtm/version2 1/Documentation/MIL-PDF89020B.pdf

[18] HO, K., LU, X., KOVAVISARUCH, L. Source localization using TDOA and FDOA measurements in the presence of receiver location errors: analysis and solution. IEEE Transactions on Signal Processing, 2007, vol. 55, no. 2, p. 684-696. DOI: 10.1109/TSP.2006.885744

[19] WANG, G., LI, Y., ANSARI, N. A semidefinite relaxation method for source localization using TDOA and FDOA measurements, IEEE Transactions on Vehicular Technology, 2013, vol. 62, no. 2, p. 853-862. DOI: 10.1109/TVT.2012.2225074

[20] LIU, Y., GUO, F., YANG, L., JIANG, W. An improved algebraic solution for TDOA localization with sensor position errors. IEEE Communications Letters, 2015, vol. 19, no. 12, p. 2218-2221. DOI: 10.1109/LCOMM.2015.2486769

[21] DALVEREN, Y., KARA, A. Comparative analysis of TDOAbased localization methods in the presence of sensor position errors. In Proceedings of the IEEE 4th International Conference on Control, Decision and Information Technologies. Barcelona (Spain), 2017, p. 556-560. DOI: 10.1109/CoDIT.2017.8102652

[22] TRIVEDI, K. S. Probability and Statistics with Reliability, Queuing and Computer Science Applications. $2^{\text {nd }}$ ed., New York (USA): John Wiley \& Sons, 2002. ISBN: 9781119285427

[23] DALVEREN, Y., KARA, A. An approach for identifying the likelihood of an irregular terrain profile being a multipath scattering center in emitter localization. In Proceedings of the $7^{\text {th }}$ International Conference on Information Communication and Management. Moscow (Russia), 2017, p. 36-39. DOI: $10.1145 / 3134383.3134402$

\section{About the Authors ...}

Yaser DALVEREN received his B.Sc., M.Sc., and Ph.D. degree from Atilim University in 2009, 2011, and 2016, respectively. His research interests include source localization, signal processing and wireless communications. He is currently serving as an Assistant Professor in the Department of Avionics at Atilim University.

Ali KARA was born in Amasya, Turkey, in 1972. He received Electronics Engineering degree from Erciyes University, Kayseri, in 1992, the MSc degree from Cukurova University, Adana, 1996, and Ph.D. degree from Hacettepe University, Ankara, 2002. He was with Polytechnic Uni- 
versity (ECE), Brooklyn, NY, between 1999 and 2000, where he conducted theoretical and experimental research as a research assistant in a project sponsored by Symbol Technologies (Motorola). He joined the Department of Electrical and Electronics Engineering at Atilim University in 2000, and devoted himself to founding the Department. He has published in refereed journals/conferences, and has led several projects in the areas of Radio Propagation, Virtual and Remote Laboratories (VRL) and Radar \& Electronic Warfare Systems. In one of the projects, he led a group at Atilim University to develop a VRL platform on
RF and Communications (unique platform funded by European Commission). On the other hand, he was with TUBITAK-BILGEM, as a chief researcher and consultant on defense and security related projects, between 2006 and 2012. He is currently with Atilim University where he has been professor since 2015, and also the Director of Graduate School of Natural and Applied Sciences. He is actively researching in areas of RF fingerprinting, locating and identification of radio emitters including radars and wireless devices, radio aspects of wireless communications including channel modeling and antennas. 